\title{
Allergy to beta-lactams in pediatrics: a practical approach
}

\author{
Nelson A. Rosário, ${ }^{1}$ Anete Sevciovic Grumach ${ }^{2}$
}

\begin{abstract}
Objective: To present a practical approach to the diagnosis and management of allergy to beta-lactam antibiotics.

Sources: Allergy journals indexed in MEDLINE and LILACS, as well as seminal studies and texts.

Summary of the findings: Allergy to penicillin is commonly reported. In many cases, this results in the decision not to use this drug. About $10 \%$ of drug allergy reports are confirmed. The clinical manifestations due to allergic reaction to penicillin vary widely, with emphasis on skin disorders. Gell \& Coombs' four hypersensitivity mechanisms are involved in allergic reactions. Penicillin is degraded to a major (95\%) and minor determinants $(5 \%)$. Immediate IgE-mediated reactions causing anaphylaxis are associated with minor determinants in $95 \%$ of the cases. Hypersensitivity to these products can be assessed using cutaneous tests performed with major and minor determinants, thus avoiding anaphylactic shock in allergic individuals. The present article underscores the basic body of knowledge on allergy to penicillin, providing support for a more accurate diagnosis of this event and for the choice of management in cases of suspected beta-lactam allergy.

Conclusions: The incorrect diagnosis of penicillin allergy frequently leads to the exclusion of this drug as a therapeutic option. A better recognition of these situations will enable the use of penicillin and reduce the risks associated with hypersensitivity.
\end{abstract}

J Pediatr (Rio J). 2006;82(5 Suppl):S181-8: Beta-lactam, penicillin, cephalosporin, allergy, hypersensitivity reaction.

\section{Introduction}

Since penicillin was introduced as a therapeutic option, around 60 years ago, several studies on allergic reactions and possible consequences of its use have been reported ${ }^{1-3}$ (Table 1 ). Between 10 and $20 \%$ of hospitalized individuals report allergy to penicillin. This fact has often resulted in the replacement of penicillin by other drugs, sometimes jeopardizing the patient's treatment. ${ }^{4}$ Examples as congenital syphilis and rheumatic fever reinforce the need of approaching this theme based on the acquired knowledge.

Prescription and administration of penicillin have been suffering considerable reduction due to several factors, among which is fear of allergic reactions and intensive

1. Doutor. Professor titular, Universidade Federal do Paraná(UFPR), Curitiba, PR, Brasil. Coordenador, Curso de Especialização em Alergia Pediátrica, UFPR, Curitiba, PR, Brasil.

2. Doutora. Médica, Ambulatório de Imunodeficiências Primárias, Departamento de Dermatologia, Faculdade de Medicina, Universidade de São Paulo (USP), São Paulo, SP, Brasil. Pesquisadora, Laboratório de Dermatopatologia e Imunodeficiências, Departamento de Dermatologia, Faculdade de Medicina, USP, São Paulo, SP, Brasil.

Suggested citation: Rosário NA, Grumach AS. Allergy to beta-lactams in pediatrics: a practical approach. J Pediatr (Rio J). 2006;82(5 Suppl):S181-8. marketing strategies for new antibiotics. ${ }^{5-7}$ However, most people deemed allergic to penicillin may safely receive this antibiotic; this occurs because sensitivity disappears over time, or because the reaction might be mistaken by cutaneous eruption of a virosis. It could also be a reaction to another drug ingested with the antibiotic, such as antipyretic or non-hormonal anti-inflammatory drugs, usually prescribed for respiratory infection in children. 8

Penicillins are a group of antibiotics of very low cost and high efficacy; therefore, they are important in the treatment and prevention of pyogenic infectious diseases and their complications. They are first-choice antibiotics in infections caused by sensitive encapsulated agents (pneumococcal pneumonia, cerebral abscess, bacterial meningitis), in syphilis (congenital, gestational neurosyphilis associated with HIV), primary and secondary prophylaxis of rheumatic fever and in post-streptococcal glomerulonephritis.

Penicillin is thus recommended by the World Health Organization (WHO) as being essentially and undoubtedly useful. It has become stigmatized by health professionals and by the general population, mainly due to lack of 
Table 1 - Hypersensitivity reactions in beta-lactam allergies ${ }^{1}$

\begin{tabular}{ccc}
\hline $\begin{array}{c}\text { Gel-Coombs } \\
\text { classification }\end{array}$ & Mechanism & $\begin{array}{c}\text { Examples of adverse reactions } \\
\text { to penicillin }\end{array}$ \\
\hline I & $\begin{array}{c}\text { Anaphylactic (IgE-mediated) } \\
\text { Complement cytolysis (IgG/IgM) } \\
\text { Thrombocytopenia } \\
\text { Interstitial nephritis } \\
\text { II }\end{array}$ & $\begin{array}{c}\text { Acute anaphylaxis, urticaria } \\
\text { Hesion by immune-complexes } \\
\text { Drug fever }\end{array}$ \\
III & $\begin{array}{c}\text { Some rashes and vasculitis } \\
\text { IV }\end{array}$ & Serum disease \\
& Late or cell-mediated hypersensitivity \\
Morbilliform rashes & Contact dermatitis \\
\hline
\end{tabular}

updated information with regard to allergic reactions. ${ }^{7}$ Reports of reactions are frequently related to neurovegetative disorders, characterized by anxiety, fear and sweating, associated with pain or possibility of painful feeling as a response to administration of parenteral drugs or other medical procedures. These episodes are usually and wrongly interpreted as allergic reactions, especially by the general population. In such situations, there is excessive agitation and anxiety, which may cease in a few minutes only by maintaining the patient lying down, with no need of any type of specific treatment. ${ }^{1,3}$

Chance of developing an allergic reaction to penicillin is approximately $2 \%$ per treatment. Anaphylactic reactions rarely occur. A more than 3-year clinical follow-up of patients taking benzathine penicillin for prophylaxis of rheumatic disease showed a $3.2 \%$ incidence of reactions and anaphylaxis incidence in $1.23 / 10,000$ injections. Fatal reaction occurred in $1 / 32,000$ injections. 9,10 Studies have demonstrated that allergic reactions considered moderate are not very frequent, present in 0.5-1.0/1,000 treatments, and rare fatal anaphylactic reactions may occur in 1.0-2.0/ 100,000 treatments.

Hypersensitivity reactions may occur with any type of drug, which means that attention and care required for penicillin are the same given to other drugs.

\section{Which are the mechanisms involved in reaction to B-lactams?}

B-lactam antibiotics include this ring in its molecular structure and correspond to penicillin, aminopenicillin (amoxicillin), cephalosporins, carbapenems (imipenem) and monobactams (aztreonam) (Figure 1). With regard to penicillin, the ring is fused to thiazolidine (6aminopenicillanic acid). In cephalosporins, the ring is fused to 1,3-thiazine structure. Some anaphylactic reactions to cephalosporins are a consequence of antibodies directed against side chains specific in these molecules more than to the $B$-lactam ring. ${ }^{11,12}$ Therefore, allergy to penicillin does not exclude use of cephalosporins, and it is estimated that crossed reactions occur in about $7-10 \%$ of cases. ${ }^{13}$

Penicillin is a low molecular weight chemical substance and needs to bind covalently to tissue macromolecules to produce hapten-protein complexes and induce immune response. ${ }^{1,7}$ The $B$-lactam ring opens spontaneously under physiological conditions, forming a starch binding with epsilon-amino groups of adjacent proteins. Reactions to drugs occur by all mechanisms described by Gell \& Coombs. Nevertheless, more severe reactions, such as anaphylactic shock and edema of the glottis, are associated with the type I mechanism, i.e., reaction mediated by IgE antibodies. Two groups of antigens associated with adverse reactions are obtained from penicillin: benzylpenicilloyl, which is the major determinant and represents $95 \%$ of the total, and minor determinants, which represent $5 \%$, including penicilline, penilloate, penicillamine and penicillin itself. ${ }^{9}$

\section{What are the main clinical manifestations of allergy to penicillin?}

Hypersensitivity reactions to penicillin may be divided into:

a) immediate reactions, which occur up to 20 minutes after parenteral administration of penicillin and up to 1 hour after oral administration. These are generally 
the most severe reactions. They include urticaria, diffuse pruritus, skin flare and, less frequently, laryngeal edema, cardiac arrhythmia and shock. These are IgEmediated reactions; about $95 \%$ of cases are directed against minor antigenic determinants of penicillin. They account for $75 \%$ of deaths due to anaphylactic reaction in the USA. 4,5

b) Accelerated reactions, manifested between 1-72 hours after administration of penicillin. They cause urticaria or angioedema, laryngeal edema and, in rare occasions, hypotension and death. They are a consequence of antibodies against the main antigenic determinant of penicillin in $95 \%$ of cases.

c) Late reactions, which are the most common. They are seen after 72 hours, with benign, morbilliform rashes with good outcome. Less frequently, reactions are not cutaneous, such as fever, serum sickness, immune hemolytic anemia, thrombocytopenia, acute interstitial nephritis, pulmonary infiltration with eosinophilia, hypersensitivity vasculitis, drug fever, Stevens-Johnson syndrome and exfoliative dermatitis. The physiopathological mechanism is not known.

\section{How to diagnose allergy to B-lactams?}

Clinical history has been used to direct choice of antibiotic and, with regard to B-lactams, it might suggest occurrence of allergic reactions. However, it would be adequate to consider the answer to the following question as definitive: have you presented reaction to penicillin before? It is known that, to make an allergic reaction occur, there must be previous exposure to the antigen. However, sensitivity may occur in certain circumstances different from the usual administration of antibiotics and<smiles>O=C(Cc1ccccc1)N[C@H]1C(=O)N2[C@@H](C(=O)O)CS[C@H]12</smiles>

B-lactam ring

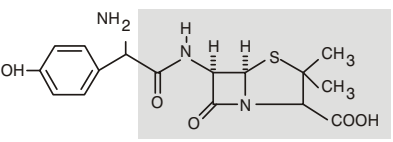

Amoxicillin

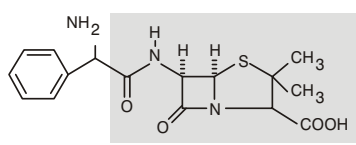

Ampicillin

\section{Cephalosporin}<smiles>[2H]CC1=C([N+](=O)[O-])N2C(=O)[C@H](NC([2H])=O)[C@H]2SC1</smiles>

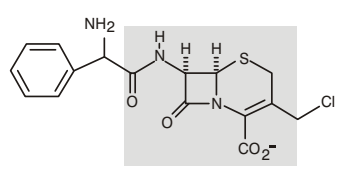

Cefaclor

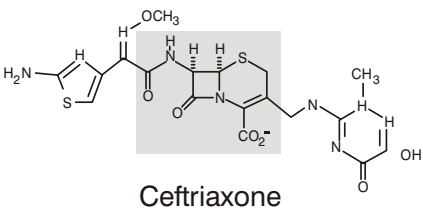

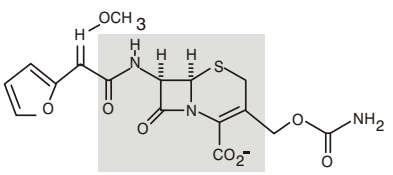

Cefuroxime

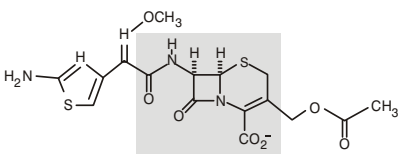

Cefotaxime

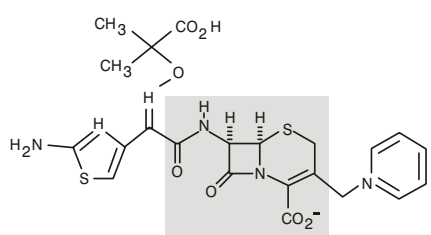

Ceftazidime

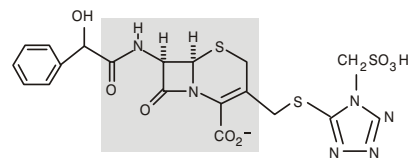

Cefonicid

Figure 1 - Structure of the beta-lactam ring and antibiotics that have this ring in their structure ${ }^{38}$ 
patients are not always aware of this relation. Therefore, penicillin particles may be inhaled in environments where this drug is manipulated; in addition, antibiotics are used in foods to avoid contamination, and some creams and ointments have antibiotics in their ingredients. Less than $10 \%$ of individuals with history of allergy to penicillin present positive skin tests. ${ }^{14}$ Therefore, reports of previous exposure to drugs are not always reliable and do not clarify how the sensitivity occurred. From $1 / 4$ to $1 / 3$ of patients who die due to allergy to penicillin presented history of previous reaction to this drug. On the other hand, only between $0.12-0.26 \%$ with positive history and tested for allergy to penicillin present systemic reactions. ${ }^{7,15}$ Therefore, clinical data may alert physicians for a possible reaction, but should not be used for treatment exclusion.

Family history of allergy to drugs is not associated with higher frequency of reaction in individuals. Also, allergic individuals are not more prone to allergy to penicillin, but when there are reactions, these may be more intense. Adkinson et al. ${ }^{1}$ demonstrated formation of IgE antibodies against penicillin in $18 \%$ of volunteers exposed to this drug.

Personal history may be used for screening individuals likely to have allergic reactions to drugs; for example, whether there is association with infection by HIV, EpsteinBarr virus and cytomegalovirus, or lymphomas and leukemias. Reactions occur more frequently in women, aged between 20-49 years and are less common in children (Table 2 ).

Besides clinical history data, diagnosis of hypersensitivity reactions using skin tests may confirm the suspicion. ${ }^{16-19}$ It is known that minor determinants are the main triggering factors of IgE-mediated reactions, i.e., type I, so a mixture of these determinants has been used for skin tests. It is a simple procedure that could predict about $95 \%$ of severe reactions. This test should be performed immediately after administering the drug, since its predictive value is limited to the period previous to penicillin use. In addition, performing allergic tests may sensitize individuals. ${ }^{7}$

\section{How to perform allergic tests to diagnose allergy to B-lactams?}

To date, performing allergic tests to penicillin is still a controversial issue. It is known that their use allows the identification of allergic individuals. However, this issue has been discussed due to traditional use of the commercial product itself, which is inadequate for performing the test. Diagnosis of allergy to penicillin and administration of this drug were traditionally dependent on performing the intradermal test with benzathine penicillin, an inappropriate form of assessing allergic response to the product. This test resulted in non-specific reactions to the drug, or it could trigger anaphylactic shock due to drug exposure in environments that are not prepared for emergency services. Therefore, by choosing to perform an allergic test, the adequate product must be used. It should be stressed that the tests are only useful for reactions that involve IgE antibodies. They have no predictive value for reactions such as late exanthema, fever, hemolytic anemia, exfoliative dermatitis, Stevens-Johnson syndrome, contact dermatitis, interstitial nephritis or serum disease. 8,20

Between $10-20 \%$ of hospitalized patients report previous history of allergy to penicillin. However, most present negative skin tests, and only about 3\% develop reaction if submitted to penicillin provocation test. ${ }^{3,14}$ In practice, despite the availability of tests, most patients receive another antibiotic agent to replace penicillin. ${ }^{15}$

Guidelines to perform skin tests have been published by the EACCI Interest Group on Drug Hypersentivity. ${ }^{17}$ Their suggestion is to use haptenic reagents of major determinant (PPL) and minor determinants (MDM), which are not available in several countries, including Brazil. 19,20 Skin tests represent the best way to avoid the unnecessary label of "allergic to penicillin." Bousquet et al. assessed 824 patients during 8 years as to allergy to ß-lactams,

Table 2 - Facts and myths that influence exclusion of beta-lactams

\begin{tabular}{ll}
\hline Atopy is a risk factor & (myth) \\
Sensitivity to fungi is related to allergic reactions & (myth) \\
Intravenous administration offers more risk & (fact) \\
Common in any age group & (more common in adults) \\
History of previous reaction & (6-fold increase in risk) \\
Allergy to penicillin excludes use of cephalosporin & (myth)
\end{tabular}


performing tests with the antibiotics reported as causing the reaction, besides testing benzylpenicilloyl polylysine (PPL, main determinant) and minor determinant mixture (MDM). The patients who presented positive tests were not provoked, and 20 of them only reacted to PPL and/or MDM. The percentage of positive reactions was small, but if those reagents were not available, the individuals would have been submitted to provocation unnecessarily. Also, of the 688 negative tests, $53(7.7 \%)$ patients developed reaction to oral provocation, including anaphylactic shock in 11.21

False-positive and false-negative skin tests are unusual, but may occur. Incidence of positive skin tests among patients with negative history (false-positive) is low, around $7 \%$. Incidence of accelerated or immediate reactions after administration of penicillin in patients with positive history and negative skin tests (false-negative) is probably lower than $1 \%$. When patients with positive history and skin tests are given penicillin, incidence of significant allergic reactions is between $65-73 \%$ (Table 3 ). Therefore, allergic tests performed with these drugs present good predictive ability for immediate reactions. Theoretical risk of recurrent sensitivity of a patient with positive history, but negative skin test is low, about 3\%.22 Therefore, in this case, and having tolerated the B-lactam antibiotic treatment, there is no need of performing new tests (Figure 2). Patients with previous history of allergy to penicillin, with negative skin tests for penicillin, are not likely to have new reactions or recurrent sensitivity during subsequent treatments with penicillin. 22-25 Macy et al. ${ }^{22}$ reviewed medical charts of 568 individuals who were given at least one treatment with oral penicillin after negative test. These patients underwent between 1 and 22 treatments ( 4 in average) and reported reactions in 27
$(4.8 \%)$ of tested patients $(n=568)$; most of them developed rashes, but no severe reaction. These data confirm previous findings in children. $24-27$

Diagnosis of allergy to penicillin should be adequately evaluated before excluding it from medical prescription. Such adequate evaluation comprehends, in the first place, previous inquiry to guide towards a real need of indicating penicillin sensitivity tests, which are not necessary in most cases. 28,29

These tests should only be performed with adequate preparations. Penicillin should never be given using the intradermal approach or any other approach, since such procedure may cause immediate anaphylactic reaction.

\section{Is the access to material for allergic tests difficult? Is it dangerous to perform the tests?}

Considering what was previously mentioned, skin tests with minor determinants are more appropriate to prevent most severe reactions caused by penicillin. Preparation of a mixture of minor determinants can be performed in

Table 3 - Predictive value of clinical history and skin tests for risk of allergic reaction

\begin{tabular}{lcc}
\hline Clinical history & Skin tests & Risk \\
\hline Positive & Negative & $<3 \%$ \\
Positive & Positive & $50 \%$ \\
Positive & Negative & Minimum for cephalosporin \\
Positive & Positive & $10 \%$ for cephalosporin \\
\hline
\end{tabular}

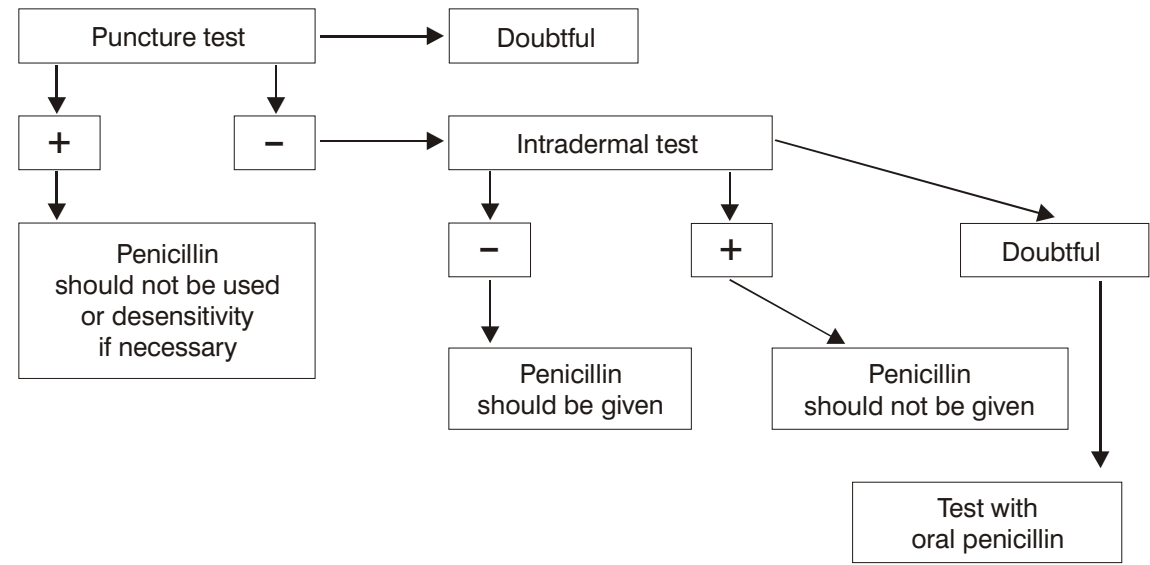

Figure 2 - Practical guidelines for diagnosing allergy to penicillin 
services that have a laboratory, by treating penicillin G potassium with a solution of sodium hydroxide $0.1 \mathrm{~N}$. On the other hand, one can choose to perform the test with a solution of 10,000 units/mL of penicillin G (P.G.) as an alternative to MDM. This solution containing penicillin $\mathrm{G}$ potassium $10,000 \mathrm{U} / \mathrm{mL}$ expires in 24 hours under refrigeration. For cases of extreme sensitivity and with history of severe reaction, it is recommended to use higher penicillin dilutions and gradually increase until $10,000 \mathrm{U}$. Skin prick tests should precede intradermal tests in these cases. The major determinant is commercially available, but its cost is high. 1,7

In our country, Sarti published his experience with skin tests for penicillin in 1985, assessing 6,764 individuals and reporting occurrence of markedly reduced positivity in children. It is important to reinforce that risk of severe systemic side effects to the prick test is extremely low. Therefore, if the procedure is adequately performed, it is possible to identify people with hypersensitivity to penicillin with reasonable safety. ${ }^{1,5,6}$ Similarly to other allergic tests, before performing it, one should check whether the patient is taking drugs such as antihistamines and oral decongestants that interfere with the allergic response.

\section{Diagnosis of allergy was confirmed. What about desensitization?}

After a diagnosis of allergy to penicillin has been confirmed, desensitization can be started. It is performed in a short period of time (24-48 hours), followed by drug administration. ${ }^{1,30}$ There are several protocols for drug desensitization. Nevertheless, all of them are based on progressive increase in concentrations of the drug to be administered, starting with diluted samples and ending the process with preparations using dilutions similar to those that will be used (Table 4). With regard to penicillin, desensitization is indicated when it is not possible to replace the drug. $20,21,31$

\section{Can patients who are allergic to penicillin be given cephalosporins?}

Cephalosporins may cause allergic reaction, independent of the crossed reaction, since they present specific epitopes and others in common with penicillin. This fact occurs more frequently with first-generation cephalosporins. ${ }^{11,12,32}$ Romano et al. ${ }^{13}$ described 30 patients with immediate reactions ( 25 with anaphylactic shock) to one type of cephalosporin ( $2 \mathrm{mg} / \mathrm{mL}$ ), and confirmed, using positive skin tests, sensitivity to cephalosporin in all individuals. Of the 30 patients, 22 presented negative test to penicillin. Incidence of reactions to cephalosporin is higher in individuals with history of allergy to penicillin (8.1 versus $1.9 \%) .{ }^{6}$ In
Table 4 - Example of a protocol for penicillin desensitivity

\begin{tabular}{ll}
\hline Time & Dose \\
\hline 0 min & $100 \mathrm{U}$ oral (penicillin V) \\
15 min & $200 \mathrm{U}$ oral \\
$30 \mathrm{~min}$ & $400 \mathrm{U}$ oral \\
$45 \mathrm{~min}$ & $800 \mathrm{U}$ oral \\
$1 \mathrm{~h}$ & $1,600 \mathrm{U}$ oral \\
$1 \mathrm{~h} 15$ & $3,200 \mathrm{U}$ oral \\
$1 \mathrm{~h} 30$ & $6,400 \mathrm{U}$ oral \\
$1 \mathrm{~h} 45$ & $12,800 \mathrm{U}$ oral \\
$2 \mathrm{~h}$ & $25,000 \mathrm{U}$ oral \\
$2 \mathrm{~h} 15$ & $50,000 \mathrm{U}$ oral \\
$2 \mathrm{~h} 30$ & $100,000 \mathrm{U}$ oral \\
$2 \mathrm{~h} 45$ & $200,000 \mathrm{U}$ oral \\
$3 \mathrm{~h}$ & $400,000 \mathrm{U}$ oral \\
$3 \mathrm{~h} 15$ & $200,000 \mathrm{U}$ subcutaneous (penicillin G) \\
$3 \mathrm{~h} 30$ & $400,000 \mathrm{U}$ subcutaneous \\
$3 \mathrm{~h} 45$ & $800,000 \mathrm{U}$ subcutaneous \\
$4 \mathrm{~h}$ & $1,000,000$ U intramuscular
\end{tabular}

Source: Stark et al. ${ }^{31}$

severe reactions to antibiotics, cephalosporins account

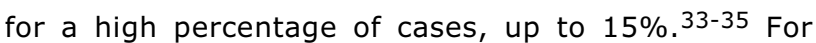
third-generation cephalosporins (e.g., ceftazidime), relative risk of reaction is lower than for first- or secondgeneration cephalosporins. In a retrospective assessment of hospitalized patients with history of allergy to penicillin (excluding severe reactions), frequency of reaction to cephalosporin was reported as $0.17 \% .36$

In case cephalosporin should be administered to individuals with allergy to penicillin, skin tests with penicillin should be previously performed, and the treatment can be introduced in negative cases. On the other hand, if the tests are positive, one can also proceed to desensitivity. ${ }^{37}$

In the past, it was common to assess tolerance to cephalosporins in patients primarily sensitized to penicillin. With the growing use of cephalosporins, there has been an inversion in facts. Antunez et al. ${ }^{38}$ assessed 24 patients with previous reaction to cephalosporin and positive RAST to this antibiotic. These authors noted that two patients also presented positive skin tests to penicillin, whereas the others $^{22}$ were negative to antigenic determinants of penicillin and tolerated parenteral administration of penicillin. The authors showed that the R1 side chain, and not the B-lactam structure, common to both drugs, had been crucial for immune recognition. Therefore, penicillin can be safely administered to patients allergic to cephalosporins and with negative tests to penicillin determinants. To administer another cephalosporin, it is recommended to select a drug with different R1 side chain. 
The group of carbapenems, represented by imipenem, presents extensive crossed reactivity of its metabolites with those of penicillin. Sodhi et al. ${ }^{39}$ registered occurrence of $9.2 \%$ reaction to carbapenem in 163 patients with history of allergy to penicillin, and such frequency was $3.9 \%$ in patients with no history of allergy to penicillin. 39

The group of monobactams, whose prototype is aztreonam, seems to be little immunogenic, with low crossed reactivity. For this reason, it has been well tolerated by individuals allergic to other B-lactam antibiotics. ${ }^{7}$

\section{Conclusion}

Skin tests with penicillin play a major role in reducing unnecessary use of wide-spectrum antibiotics in patients labeled as allergic to penicillin, thus resulting in lower development of bacterial resistance. Specialists must inform pediatricians about the option of skin tests to penicillin, since most patients do not present IgEspecific antibodies and may be given the drug with safety. ${ }^{40}$ Even if diagnostic methods cannot be applied, it is important for the pediatrician to know how to manage risks resulting from drug administration and available resources to identify possible side effects.

\section{References}

1. Adkinson NF Jr. Drug Allergy. In: Middleton's allergy: principles and practice. 6th ed. Saint Louis: Elsevier; 2006.

2. Nicklas RA. Beta-lactam antibiotics. J Allergy Clin Immunol. 1998;101:S498-501.

3. Malamann MF, Adkinson NF. B-lactams. In: Honsinger RW, Green GR, eds. Handbook of drug allergy. Philadelphia, PA: Williams \& Wilkins; 2004.

4. Saxon J. Immediate hypersensitivity reactions to B-lactam antibiotics. Ann Intern Med. 1987;107:204-15.

5. Weiss ME, Adkinson NF. Immediate hypersensitivity reactions to penicillin and related antibiotics. Clin Allergy. 1988; 18:515-8.

6. Lyn RY. A perspective of penicillin allergy. Arch Intern Med. $1992 ; 152: 930-7$

7. Sarti W. Alergia a drogas. In: Alergia e imunologia na infância e na adolescência. Grumach AS, ed. São Paulo: Atheneu; 2001.

8. Mendelson LM. Adverse reactions to B-lactam antibiotics. Immunol Allergy Clin N Am. 1998;18:745-57.

9. Greenberger PA. Drug allergy. J Allergy Clin Immunol. 2006;117: S464-70.

10. Markowitz M, Lue HC. Allergic reactions in rheumatic fever patients on long-term benzathine penicillin $\mathrm{G}$ : the role of skin testing for penicillin allergy. Pediatrics. 1996:97:981-3.

11. Romano A, Quaratino D, Aimone-Gastin I, Mayorga C, Papa G, Venuti $A$, et al. Cefalosporin allergy: characterization of unique and cross-reacting cephalosporin antigens. Int J Immunopathol Pharmacol. 1997;10:187-91.

12. Romano A, Mayorga C, Torres MJ, Artesani MC, Suau R, Perez E, et al. Immediate allergic reactions to cephalosporins: crossreactivity and selective responses. J Allergy Clin Immunol. 2000;106:1177-83.
13. Romano A, Gueant-Rodriguez RM, Viola M, Pettinato R, Gueant JL. Cross-reactivity and tolerability of cephalosporins in patients with immediate hypersensitivity to penicillins. Ann Intern Med. 2004;141:16-22.

14. Arroliga ME, Wagner W, Bobek MB, Hoffman-Hogg L, Gordon SM, Arroliga AC. A pilot study of penicillin skin testing in patients with a history of penicillin allergy admitted to a medical ICU. Chest. 2000;118:1106-8

15. Nadarajah K, Green GR, Naglak M. Clinical outcomes of penicillin skin testing. Ann Allergy Asthma Immunol. 2005;95:541-5.

16. Redelmeier DA, Sox HC Jr. The role of skin testing for penicillin allergy. Arch Intern Med. 1990;150:1939-45.

17. Torres MJ, Blanca M, Fernandez J, Romano A, Weck A, Aberer W, et al. Diagnosis of immediate allergic reactions to beta-lactam antibiotics. Allergy. 2003;58:961-72.

18. Brockow K, Romano A, Blanca M, Ring J, Pichler W, Demoly P. General considerations for skin test procedures in the diagnosis of drug hypersensitivity. Allergy. 2002;57:45-51.

19. Torres MJ, Romano A, Mayorga C, Moya MC, Guzman AE, Reche $M$, et al. Diagnostic evaluation of a large group of patients with immediate allergy to penicillins: the role of skin testing. Allergy. 2001;56:850-6.

20. Messaad D, Sahla H, Benahmed S, Godard P, Bousquet J, Demoly $P$. Drug provocation tests in patients with a history suggesting an immediate drug hypersensitivity reaction. Ann Intern Med. 2004;140:1001-6.

21. Bousquet PJ, Co-Minh HB, Amoux B, Daures JP, Demoly P. Importance of minor determinants and benzylpenicilloyl poly-LLysine skin testing in the diagnosis of B-lactam allergy. J Allergy Clin Immunol. 2005;115:1314-6.

22. Macy E, Mangat R, Burchette RJ. Penicillin skin testing in advance of need: multiyear follow-up in 568 test-result negative subjects exposed to oral penicillins. J Allergy Clin Immunol. 2003;111:1111-5

23. Sicherer $\mathrm{SH}$, Leung DY. Advances in allergic skin disease, anaphylaxis, and hypersensitivity reactions to foods, drugs, and insect stings. J Allergy Clin Immunol. 2004;114:118-24.

24. Parker PJ, Parrinello JT, Condemi J, Rose SI. Penicillin resensitization among hospitalized patients. J Allergy Clin Immunol. 1991;88:213-7.

25. Pichichero ME, Pichichero DM. Diagnosis of penicillin, amoxicillin and cephalosporin allergy: reliability of examination assessed by skin testing and oral challenge. J Pediatr. 1998;132:137-43.

26. Mendelson LM, Ressler C, Rosen JP, Selcow JE. Routine elective penicillin allergy skin testing in children and adolescents: study of sensitization. J Allergy Clin Immunol. 1984;73:76-81.

27. Solensky R, Earl HS, Gruchalla RS. Lack of penicillin resensitization in patients with a history of penicillin allergy after receiving repeated penicillin courses. Arch Intern Med. 2002;162:822-6.

28. Salkind $A R$, Cuddy $P G$, Foxworth JW. The rational clinical examination: is this patient allergic to penicillin? An evidencebased analysis of the likelihood of penicillin allergy. JAMA. 2001:285:2498-505.

29. Sogn DD, Evans R III, Shepherd GM, Casale T, Condemi J, Greenberger $P$, et al. Results of the National Institute of Allergy and Infectious Diseases Collaborative Clinical Trial to test the predictive value of skin testing with major and minor penicillin derivatives in hospitalized adults. Arch Intern Med. 1992;152:102-32

30. Stark BJ, Earl HS, Gross GN, Lumry WR, Goodman EL, Sullivan TJ. Acute and chronic desensitization of penicillin allergic patients using oral penicillin. J Allergy Clin. Immunol. 1987;79:523-32.

31. Sarti W. Routine use of skin testing for immediate penicillin allergy to 6764 patients in an outpatient clinic. Ann Allergy. 1985;55:157-61.

32. Miranda A, Blanca M, Vega JM, Moreno F, Carmona MJ, Garcia JJ, et al. Cross-reactivity between a penicillin and a cephalosporin with the same side chain. J Allergy Clin Immunol. 1996;98:671-7.

33. Kelkar PS, Li JT. Cephalosporin allergy. N Engl J Med. 2001;345: 804-9.

34. Empedrad R, Darter AL, Earl HS, Gruchalla RS. Nonirritating intradermal skin test concentrations for commonly prescribed antibiotics. J Allergy Clin Immunol. 2003;112:629-30.

35. Kanny G, Guenard L, Demoly P, Ponvert C, Grand J, Gallen C, et al. Severe drug allergy: the first 100 cases declared to Allergy Vigilance Network. J Allergy Clin Immunol. 2005;115:S183.

36. Daulat S, Solensky R, Earl HS, Casey W, Gruchalla RS. Safety of cephalosporin administration to patients with histories of penicillin allergy. J Allergy Clin Immunol. 2004;113:1220-2. 
37. Win $\mathrm{PH}$, Brown $\mathrm{H}$, Zankar A, Ballas ZK, Hussain I. Rapid intravenous cephalosporin desensitization. J Allergy Clin Immunol. 2005;116:225-8.

38. Antunez C, Blanca-Lopez N, Torres MJ, Mayorga C, PerezInestrosa $\mathrm{E}$, Montanez MI, Fernandez T, Blanca M. Immediate allergic reactions to cephalosporins: evaluation of cross-reactivity with a panel of penicillins and cephalosporins. J Allergy Clin Immunol. 2006;117:404-10.

39. Sodhi M, Axtell SS, Callahan J, Shekar R. Is it safe to use carbapenems in patients with a history of allergy to penicillin? J Antimicrob Chemother. 2004;54:1155-7.
40. Solensky R, Earl HS, Gruchalla RS. Penicillin allergy: prevalence of vague history in skin test-positive patients. Ann Allergy Asthma Immunol. 2000;85:195-9.

Correspondence:

Nelson Rosário

Hospital de Clínicas UFPR

Rua General Carneiro, 181

CEP 80060-900 - Curitiba, PR - Brazil

Tel.: +55 (41) 3360.1800

E-mail: nelson.rosario@ufpr.br 